\title{
Generation of a stochastic precipitation model for the tropical climate
}

\begin{abstract}
A tropical country like Malaysia is characterized by intense localized precipitation with temperatures remaining relatively constant throughout the year. A stochastic modeling of precipitation in the flood-prone Kelantan River Basin is particularly challenging due to the high intermittency of precipitation events of the northeast monsoons. There is an urgent need to have long series of precipitation in modeling the hydrological responses. A single-site stochastic precipitation model that includes precipitation occurrence and an intensity model was developed, calibrated, and validated for the Kelantan River Basin. The simulation process was carried out separately for each station without considering the spatial correlation of precipitation. The Markov chains up to the fifthorder and six distributions were considered. The daily precipitation data of 17 rainfall stations for the study period of 1954-2013 were selected. The results suggested that second- and third-order Markov chains were suitable for simulating monthly and yearly precipitation occurrences, respectively. The fifth-order Markov chain resulted in overestimation of precipitation occurrences. For the mean, distribution, and standard deviation of precipitation amounts, the exponential, gamma, log-normal, skew normal, mixed exponential, and generalized Pareto distributions performed superiorly. However, for the extremes of precipitation, the exponential and log-normal distributions were better while the skew normal and generalized Pareto distributions tend to show underestimations. The log-normal distribution was chosen as the best distribution to simulate precipitation amounts. Overall, the stochastic precipitation model developed is considered a convenient tool to simulate the characteristics of precipitation in the Kelantan River Basin.
\end{abstract}

Keyword: Precipitation model; Kelantan River Basin; Flood; Tropical climate 
